\title{
Improving Primary Care Fall Risk Management: Adoption of Practice Changes After a Geriatric Mini-Fellowship
}

\author{
Colleen M. Casey, PhD, ANP-BC, Jamie M. Caulley, DPT, Angela F. Fox, BA,
} and Marian O. Hodges, MD, MPH

\section{ABSTRACT}

Background: Approximately 51 million adults in the United States are 65 years of age or older, yet few geriatric-trained primary care providers (PCP) serve this population. The AgeFriendly Health System framework, consisting of evidencebased 4M care (Mobility, Medication, Mentation, and what Matters), encourages all PCPs to assess mobility in older adults.

Objective: To improve PCP knowledge, confidence, and clinical practice in assessing and managing fall risk.

Methods: A 1-week educational session focusing on mobility (part of a 4-week Geriatric Mini-Fellowship) for 6 selected PCPs from a large health care system was conducted to increase knowledge and ability to address fall risk in older adults. The week included learning and practicing a Fall Risk Management Plan (FRMP) algorithm, including planning for their own practice changes. Pre- and post-test surveys assessed changes in knowledge and confidence. Patient data were compared 12 months before and after training to evaluate PCP adoption of FRMP components.

Results: The training increased provider knowledge and confidence. The trained PCPs were 1.7 times more likely to screen for fall risk; 3.6 times more likely to discuss fall risk; and 5.8 times more likely to assess orthostatic blood pressure in their 65+ patients after the mini-fellowship. In high-risk patients, they were 4.1 times more likely to discuss fall risk and 6.3 times more likely to assess orthostatic blood pressure than their nontrained peers. Changes in physical therapy referral rates were not observed.

Conclusions: In-depth, skills-based geriatric educational sessions improved PCPs' knowledge and confidence and also improved their fall risk management practices for their older patients.

Keywords: geriatrics; guidelines; Age-Friendly Health System; 4M; workforce training; practice change; fellowship.

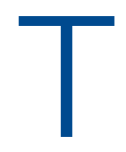

he US population is aging rapidly. People aged 85 years and older are the largest-growing segment of the US population, and this segment is expected to increase by $123 \%$ by $2040 .{ }^{1}$ Caregiving needs increase with age as older adults develop more chronic conditions, such as hypertension, heart disease, arthritis, and dementia. However, even with increasing morbidity and dependence, a majority of older adults still live in the community rather than in institutional settings. ${ }^{2}$ These older adults seek medical care more frequently than younger people, with about $22 \%$ of patients 75 years and older having 10 or more health care visits in the previous 12 months. By 2040, nearly a quarter of the US population is expected to be 65 or older, with many of these older adults seeking regular primary care from providers who do not have formal training in the care of a population with multiple complex, chronic health conditions and increased caregiving needs. ${ }^{1}$

Despite this growing demand for health care professionals trained in the care of older adults, access to these types of clinicians is limited. In 2018, there were roughly 7000 certified geriatricians, with only 3600 of them practicing full-time. ${ }^{3,4}$ Similarly, of 290,000 certified nurse practitioners (NPs), about $9 \%$ of them have geriatric certification. ${ }^{5}$ Geriatricians, medical doctors trained in the

From the Senior Health Program, Providence Health \& Services, Oregon, Portland, OR. 
care of older adults, and geriatric-trained NPs are part of a cadre of a geriatric-trained workforce that provides unique expertise in caring for older adults with chronic and advanced illness. They know how to manage multiple, complex geriatric syndromes like falls, dementia, and polypharmacy; understand and maximize team-based care; and focus on caring for an older person with a goal-centered versus a disease-centered approach. ${ }^{6}$

Broadly, geriatric care includes a spectrum of adults, from those who are aging healthfully to those who are the frailest. Research has suggested that approximately $30 \%$ of older adults need care by a geriatric-trained clinician, with the oldest and frailest patients needing more clinician time for assessment and treatment, care coordination, and coaching of caregivers. ${ }^{7}$ With this assumption in mind, it is projected that by 2025 , there will be a national shortage of 26,980 geriatricians, with the western United States disproportionately affected by this shortage. ${ }^{4}$

Rather than lamenting this shortage, Tinetti recommends a new path forward: "Our mission should not be to train enough geriatricians to provide direct care, but rather to ensure that every clinician caring for older adults is competent in geriatric principles and practices." Sometimes called "geriatricizing," the idea is to use existing geriatric providers as a small elite training force to infuse geriatric principles and skills across their colleagues in primary care and other disciplines., ${ }^{8,9}$ Efforts of the American Geriatrics Society (AGS), with support from the John A. Hartford Foundation (JAHF), have been successful in developing geriatric training across multiple specialties, including surgery, orthopedics, and emergency medicine (www.americangeriatrics.org/programs/ geriatrics-specialists-initiative).

\section{The Age-Friendly Health System and 4M Model}

To help augment this idea of equipping health care systems and their clinicians with more readily available geriatric knowledge, skills, and tools, the JAHF, along with the Institute for Healthcare Improvement $(\mathrm{IHI})$, created the Age-Friendly Health System (AFHS) paradigm in 2015. ${ }^{10}$ Using the $4 \mathrm{M}$ model, the AFHS initiative established a set of evidence-based geriatric priorities and interventions meant to improve the care of older adults, reduce harm and duplication, and provide a framework for engaging leadership, clinical teams, and operational systems across inpatient and ambulatory settings. ${ }^{11}$ Mobility, including fall risk screening and intervention, is 1 of the $4 \mathrm{M}$ foundational elements of the Age-Friendly model. In addition to Mobility, the $4 \mathrm{M}$ model also includes 3 other key geriatric domains: Mentation (dementia, depression, and delirium), Medication (high-risk medications, polypharmacy, and deprescribing), and What Matters (goals of care conversations and understanding quality of life for older patients). ${ }^{11}$ The $4 \mathrm{M}$ initiative encourages adoption of a geriatric lens that looks across chronic conditions and accounts for the interplay among geriatric syndromes, such as falls, cognitive impairment, and frailty, in order to provide care better tailored to what the patient needs and desires. ${ }^{12} \mathrm{HI}$ and JAHF have targeted the adoption of the $4 \mathrm{M}$ model by $20 \%$ of US health care systems by $2020 .{ }^{11}$

\section{Mini-Fellowship and Mobility Week}

To bolster geriatric skills among community-based primary care providers (PCPs), we initiated a Geriatric MiniFellowship, a 4-week condensed curriculum taught over 6 months. Each week focuses on 1 of the age-friendly $4 \mathrm{Ms}$, with the goal of increasing the knowledge, selfefficacy, skills, and competencies of the participating PCPs (called "fellow" hereafter) and at the same time, equipping each to become a champion of geriatric practice. This article focuses on the Mobility week, the second week of the mini-fellowship, and the effect of the week on the fellows' practice changes.

To construct the Mobility week's curriculum with a focus on the ambulatory setting, we relied upon national evidence-based work in fall risk management. The Centers for Disease Control and Prevention (CDC) has made fall risk screening and management in primary care a high priority. Using the clinical practice guidelines for managing fall risk developed by the American and British Geriatrics Societies (AGS/BGS), the CDC developed the Stopping Elderly Accidents, Deaths, and Injuries (STEADI) toolkit.13 Foundational to the toolkit is the validated 12-item Stay Independent falls screening questionnaire (STEADI questionnaire). ${ }^{14}$ Patients who score 4 or higher (out of a total score of 14) on the questionnaire are considered at increased risk of falling. The CDC has 
developed a clinical algorithm that guides clinical teams through screening and assessment to help identify appropriate interventions to target specific risk factors. Research has clearly established that a multifactorial approach to fall risk intervention can be successful in reducing fall risk by as much as $25 \% .^{15-17}$

The significant morbidity and mortality caused by falls make training nongeriatrician clinicians on how to better address fall risk imperative. More than $25 \%$ of older adults fall each year. ${ }^{18}$ These falls contribute to rising rates of fall-related deaths, ${ }^{19}$ emergency department (ED) visits, ${ }^{20}$ and hospital readmissions. ${ }^{21}$ Initiatives like the AFHS focus on mobility and the CDC's development of supporting clinical materials ${ }^{22}$ aim to improve primary care adoption of fall risk screening and intervention practices. ${ }^{23,24}$ The epidemic of falls must compel all PCPs, not just those practicing geriatrics, to make discussing and addressing fall risk and falls a priority.

\section{Methods}

\section{Setting}

This project took place as part of a regional primary care effort in Oregon. Providence Health \& Services-Oregon is part of a multi-state integrated health care system in the western United States whose PCPs serve more than 80,000 patients aged 65 years and older per year; these patients comprise $38 \%$ of the system's office visits each year. Regionally, there are 47 family and internal medicine clinics employing roughly 290 providers (physicians, NPs, and physician assistants). The organization has only 4 PCPs trained in geriatrics and does not offer any geriatric clinical consultation services. Six PCPs from different clinics, representing both rural and urban settings, are chosen to participate in the geriatric mini-fellowship each year.

This project was conducted as a quality improvement initiative within the organization and did not constitute human subjects research. It was not conducted under the oversight of the Institutional Review Board.

\section{Intervention}

The mini-fellowship was taught in 4 1-week blocks between April and October 2018, with a curriculum designed to be interactive and practical. The faculty was intentionally interdisciplinary to teach and model teambased practice. Each week participants were excused from their clinical practice. Approximately 160 hours of continuing medical education credits were awarded for the full mini-fellowship. As part of each weekly session, a performance improvement project (PIP) focused on that week's topic ( 1 of the 4Ms) was developed by the fellow and their team members to incorporate the minifellowship learnings into their clinic workflows. Fellows also had 2 hours per week of dedicated administration time for a year, outside the fellowship, to work on their PIP and $4 \mathrm{M}$ practice changes within their clinic.

\section{Provider Education}

The week for mobility training comprised 4 daylong sessions. The first 2 days were spent learning about the epidemiology of falls; risk factors for falling; how to conduct a thorough history and assessment of fall risk; and how to create a prioritized Fall Risk Management Plan (FRMP) to decrease a patient's individual fall risk through tailored interventions. The FRMP was adapted from the CDC STEADI toolkit.13 Core faculty were 2 geriatric-trained providers (NP and physician) and a physical therapist (PT) specializing in fall prevention.

On the third day, fellows took part in a simulated fall risk clinic, in which older adults volunteered to be patient partners, providing an opportunity to apply learnings from days 1 and 2. The clinic included the fellow observing a PT complete a mobility assessment and a pharmacist conduct a high-risk medication review. The fellow synthesized the findings of the mobility assessment and medication review, as well as their own history and assessment, to create a summary of fall risk recommendations to discuss with their volunteer patient partner. The fellows were observed and evaluated in their skills by their patient partner, course faculty, and another fellow. The patient partners, and their assigned fellow, also participated in a 45-minute fall risk presentation, led by a nurse.

On the fourth day, the fellows were joined by select clinic partners, including nurses, pharmacists, and/or medical assistants. The session included discussions among each fellow's clinical team regarding the current state of fall risk efforts at their clinic, an analysis of 


\section{Providence Senior Health}

\begin{tabular}{|c|c|c|}
\hline \multicolumn{3}{|c|}{$\begin{array}{c}\text { Annual STEADI (Fall Risk) Screening } \\
\text { Patients Age } 65 \& \text { Over }\end{array}$} \\
\hline Step & Tasks & Tools/Next Steps \\
\hline \multicolumn{3}{|c|}{ Pre-Visit (day prior or day of) } \\
\hline \multirow[t]{3}{*}{1} & $\begin{array}{l}\text { Chart Scrub to see if patient has any of the } \\
\text { following triggers for Fall Risk: } \\
\text { - No STEADI* screening in last } 12 \text { months } \\
\text { - Visit is for Acute Fall } \\
\text { - Post Hospital Discharge visit } \\
\text { - Recent fall or "near fall"** history }\end{array}$ & $\begin{array}{l}\text { Use Pre-Visit Chart Prep in EHR or per clinic } \\
\text { protocol. } \\
\text { *-Stopping Elderly Accidents, Deaths, and Injuries } \\
\text { **_"Near Fall" is defined as any change of position } \\
\text { to a lower level that was not intended (World } \\
\text { Health Organization). }\end{array}$ \\
\hline & If "yes" to any of the above triggers. & $\begin{array}{l}\text { Communicate STEADI is due per clinic protocol. } \\
\text { Proceed to Step } 2 \text {. }\end{array}$ \\
\hline & If 'no" to all of the above triggers. & No further action. \\
\hline
\end{tabular}

Office Visit

\begin{tabular}{|c|c|c|}
\hline 2 & $\begin{array}{l}\text { CHECK IN or ROOMING IN } \\
\text { - Ask patient to answer first } 3 \text { STEADI questions } \\
\text { of STEADI questionnaire. } \\
\text { - Review responses to first } 3 \text { STEADI questions } \\
\text { - If patient answers "yes" to any of first three } \\
\text { questions full questionnaire should be } \\
\text { completed. May ask questions verbally. }\end{array}$ & $\begin{array}{l}\text { Use STEADI flowsheet to record and use check-in } \\
\text { note to document STEADI score per clinic protocol. } \\
\text { The STEADI questionnaire can also be completed } \\
\text { with a patient over the phone. Flowsheet will } \\
\text { calculate STEADI score. } \\
\text { If STEADI score is } 4 \text { or more, proceed to step } \mathbf{3} \text {. } \\
\text { If score is } \mathbf{0 - 3} \text {, proceed to step } \mathbf{4} \text {. }\end{array}$ \\
\hline \multirow[t]{3}{*}{3} & $\begin{array}{l}\text { STEADI Score of } 4 \text { or more } \\
\text { - Flag patient as high risk for falling. }\end{array}$ & $\begin{array}{l}\text { Use magnet on door/computer; or place STEADI } \\
\text { screen on stool/ desk; or verbal handoff to } \\
\text { provider/RN. }\end{array}$ \\
\hline & $\begin{array}{l}\text { - Initiate Fall Risk Assessment and target } \\
\text { priority interventions: } \\
\text { ○ Gait evaluation, } \\
\text { ○ Orthostatic BP and } \\
\text { ○ High risk medication review. }\end{array}$ & $\begin{array}{l}\text { Use Fall Risk Assessment \& Management } \\
\text { Algorithm. } \\
\text { Enter "At risk for falls" (Z91.81) on Problem List } \\
\text { along with STEADI score (@REVFS(731:3)@) and } \\
\text { outline plan of care. }\end{array}$ \\
\hline & $\begin{array}{l}\text { Additional fall risk factors can be addressed at } \\
\text { a future regular Office Visit or Fall Risk } \\
\text { Assessment Visit. }\end{array}$ & Schedule follow-up visit while patient in clinic. \\
\hline 4 & $\begin{array}{l}\text { ALL PATIENTS BOTH LOW RISK (STEADI 0-3) OR } \\
\text { HIGH RISK (STEADI } 4 \text { OR MORE) } \\
\text { - Provide After Summary Visit(AVS) }\end{array}$ & $\begin{array}{l}\text { Provide patient with GEROFALLRISKAVSPRIORITY } \\
\text { or GEROFALLRISKAVSLOW ( GERO- } \\
\text { FALLRISKAVSHIGH also available) }\end{array}$ \\
\hline
\end{tabular}

Figure 1. Fall risk screening protocol. (C2019 Providence Health \& Services. 


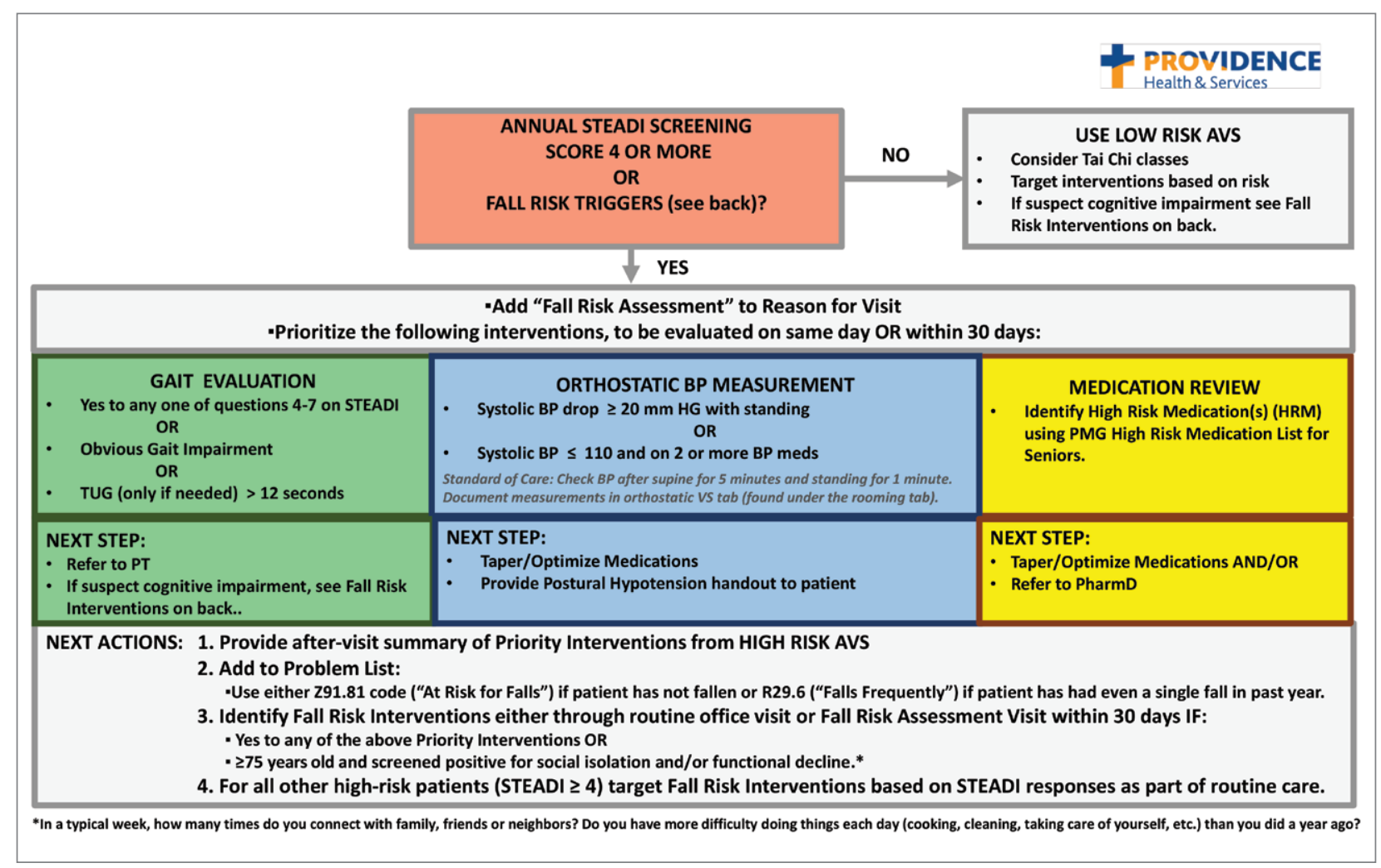

Figure 2. Fall risk assessment and intervention protocol. (C2019 Providence Health \& Services.

barriers, and identification of opportunities to improve workflows and screening rates. Each fellow took with them an action plan tailored to their clinic to improve fall risk management practices, starting with the fellow's own practice.

\section{Fall Risk Management Plan}

The educational sessions introduced the fellows to the FRMP. The FRMP, adapted from the STEADI toolkit, includes a process for fall risk screening (Figure 1) and stratifying a patient's risk based on their STEADI score in order to promote 3 priority assessments (gait evaluation with PT referral if appropriate; orthostatic blood pressure; and high-risk medication review; Figure 2). Initial actions based on these priority assessments were followed over time, with additional fall risk interventions added as clinically indicated..$^{25}$ The FRMP is intended to be used during routine office visits, Medicare annual wellness visits, or office visits focused on fall risk or related medical disorders (ie, fall risk visits.)
Providers and their teams were encouraged to spread out fall-related conversations with their patients over multiple visits, since many patients have multiple fall risk factors at play, in addition to other chronic medical issues, and since many interventions often require behavior changes on the part of the patient. Providers also had access to fall-related electronic health record (EHR) templates as well as a comprehensive, internal fall risk management website that included assessment tools, evidence-based resources, and patient handouts.

\section{Assessment and Measurements}

We assessed provider knowledge and comfort in their fall risk evaluation and management skills before and after the educational intervention using an 11-item multiple-choice questionnaire and a 4-item confidence questionnaire. The confidence questions used a 7-point Likert scale, with 0 indicating "no confidence" and 7 indicating "lots of confidence." The questions were administered via a paper survey. Qualitative comments were 
Table 1. Practice Patterns in the 12 Months Before and After Training: All PCP Visits

\begin{tabular}{|c|c|c|c|c|c|}
\hline & Pre, n (\%) & Post, n (\%) & $\mathrm{X}^{2}$ & $P$ Value & RR (95\% Cl) \\
\hline \multicolumn{6}{|l|}{ PCP visits } \\
\hline Trained providers & 3579 & 3804 & & & \\
\hline Control & 80,716 & 103,018 & & & \\
\hline \multicolumn{6}{|c|}{ Screened for fall risk using STEADI } \\
\hline Trained providers $(n=7383$ ) & 479 (13.38) & $856(22.50)$ & 103.5 & $<0.001$ & $1.681(1.52-1.86)$ \\
\hline Control $(n=183,734)$ & $17,717(21.95)$ & $24,942(24.21)$ & 129.8 & $<0.001$ & $1.103(1.08-1.12)$ \\
\hline \multicolumn{6}{|l|}{ Fall risk visits } \\
\hline Trained providers $(n=7383$ ) & $93(2.60)$ & $352(9.25)$ & 144.2 & $<0.001$ & $3.561(2.85-4.45)$ \\
\hline Control $(n=183,734)$ & $1582(1.96)$ & $2280(2.21)$ & 14.1 & $<0.001$ & $1.129(1.06-1.20)$ \\
\hline \multicolumn{6}{|c|}{ Checked orthostatic blood pressure } \\
\hline Trained providers $(n=7383$ ) & $19(0.53)$ & $118(3.10)$ & 66.9 & $<0.001$ & $5.843(3.61-9.47)$ \\
\hline Control $(n=183,734)$ & $476(0.59)$ & $681(0.66)$ & 3.7 & 0.055 & $1.121(1.00-1.26)$ \\
\hline
\end{tabular}

derived from evaluations completed at the end of the week.

The fellows' practice of fall risk screening and management was studied from May 2018, at the completion of Mobility week, to May 2019 for the post-intervention period. A 1-year timeframe before May 2018 was used as the pre-intervention period. Eligible visit types, during which we assumed fall risk was discussed, were any office visits for patients $65+$ completed by the patients' PCPs that used fall risk as a reason for the visit or had a fall-related diagnosis code. Fall risk visits performed by other clinic providers were not counted.

Of those patients who had fall risk screenings completed and were determined to be high risk (STEADI score $\geq 4$ ), data were analyzed to determine whether these patients had any fall-related follow-up visits to their PCP within 60 days of the STEADI screening. For these high-risk patients, data were studied to understand whether orthostatic blood pressure measurements were performed (as documented in a flowsheet) and whether a PT referral was placed. These data were compared with those from providers who practiced in clinics within the same system but who did not participate in the mini-fellowship. Data were obtained from the organization's EHR. Additional data were measured to evaluate patterns of deprescribing of select high-risk medications, but these data are not included in this analysis.

\section{Analysis}

A paired-samples $t$ test was used to measure changes in provider confidence levels. Data were aggregated across fellows, resulting in a mean. A chi-square test of independence was performed to examine the relationship between rates of FRMP adoption by select provider groups. Analysis included a pre- and post-intervention assessment of the fellows' adoption of FRMP practices, as well as a comparison between the fellows' practice patterns and those of a control group of PCPs in the organization's other clinics who did not participate in the mini-fellowship (nontrained control group). Excluded from the control group were providers from the same clinic as the fellows; providers in clinics with a geriatric-trained provider on staff; and clinics outside of the Portland metro and Medford service areas. We used an alpha level of 0.05 for all statistical tests. 
Table 2. Trained and Control Provider Visits in the 12 Months After Training: All PCP Visits

\begin{tabular}{|c|c|c|c|c|c|}
\hline & Trained, n (\%) & Control, n (\%) & $\mathrm{X}^{2}$ & $P$ Value & RR (95\% Cl) \\
\hline Providers & 5 & 46 & & & \\
\hline PCP visits & 3804 & 103,018 & & & \\
\hline $\begin{array}{l}\text { Screened for fall risk using STEADI } \\
(\mathrm{n}=106,822)\end{array}$ & $856(22.50)$ & 24,942 (24.21) & 5.8 & 0.016 & $0.929(0.88-0.99)$ \\
\hline Fall risk visits $(n=106,822)$ & $352(9.25)$ & $2280(2.21)$ & 756.6 & $<0.001$ & $4.181(3.75-4.66)$ \\
\hline $\begin{array}{l}\text { Checked orthostatic blood pressure } \\
(\mathrm{n}=106,822)\end{array}$ & $118(3.10)$ & $681(0.66)$ & 294.4 & $<0.001$ & $4.693(3.87-5.69)$ \\
\hline \multicolumn{6}{|c|}{$\begin{array}{l}\text { Note: This table shows trained and control (nontrained) provider practice patterns related to fall risk for older adults (age 65+) seen by their primary care provide } \\
\text { in the } 12 \text { months after the geriatric mini-fellowship training. }\end{array}$} \\
\hline
\end{tabular}

Data from 5 providers were included in the analysis of the FRMP adoption. The sixth provider changed practice settings from the clinic to the ED after completing the fellowship; her patient data were not included in the FRMP part of the analysis. EHR data included data on all visits of patients 65+, as well as data for just those $65+$ patients who had been identified as being at high risk to fall based on a STEADI score of 4 or higher.

\section{Results}

\section{Provider Questionnaire}

All 6 providers responded to the pre-intervention and post-intervention tests. For the knowledge questions, fellows, as a composite, correctly answered $57 \%$ of the questions before the intervention and $79 \%$ after the intervention. Provider confidence level in delivering fall risk care was measured prior to the training (mean, 4.12 [SD, 0.62]) and at the end of the training (mean, 6.47 [SD, $0.45]$ ), demonstrating a significant increase in confidence $(\mathrm{t}(5)=-10.46, P<0.001)$.

\section{Qualitative Comments}

Providers also had the opportunity to provide comments on their experience during the Mobility week and at the end of 1 year. In general, the simulated interdisciplinary fall risk clinic was highly rated ("the highlight of the week") as a practical strategy to embed learning principles. One fellow commented, "Putting the learning into practice helps solidify it in my brain." Fellows also appreciated the opportunity to learn and meet with their clinic colleagues to begin work on a fall-risk focused PIP and to "have a framework for what to do for people who screen positive [for fall risk]."

\section{FRMP Adoption}

A comparison of the care the fellows provided to their patients $65+$ in the 12 months pre- and post-training shows the fellows demonstrated significant changes in practice patterns. The fellows were 1.7 times more likely to screen for fall risk; 3.6 times more likely to discuss fall risk; and 5.8 times more likely to check orthostatic blood pressure than prior to the mini-fellowship (Table 1). The control providers also demonstrated significant increases in fall risk screening and discussion of fall risk between the pre- and post-intervention periods; however, the relative risk (RR) was between 1.10 and 1.13 for this group. For the control group, checking orthostatic blood pressure did not significantly change. In the 12 months after training (Table 2), the fellows were 4.2 times more likely to discuss fall risk and almost 5 times more likely to check orthostatic blood pressure than their nontrained peers for all of their patients 65+, regardless of their risk to fall.

As shown in Table 3, for those patients determined to be at high risk of falling (STEADI score $\geq 4$ ), fellows showed statistically significant increases in fall risk visits (RR, 3.02) and assessment of orthostatic blood pressure (RR, 10.68) before and after the mini-fellowship. The control providers did not show any changes in practice patterns between the pre- and post-period among patients at high risk to fall. Neither the fellows nor the control group showed changes in patterns of referral to 


\begin{tabular}{|c|c|c|c|c|c|}
\hline & Pre, n (\%) & Post, n (\%) & $\mathrm{X}^{2}$ & $P$ Value & RR (95\% Cl) \\
\hline \multicolumn{6}{|c|}{ PCP visits within 60 days of $4+$ STEADI } \\
\hline Trained providers & 219 & 564 & & & \\
\hline Control & 7032 & 10,455 & & & \\
\hline \multicolumn{6}{|l|}{ Fall risk visits } \\
\hline Trained providers $(\mathrm{n}=783$ ) & $23(10.50)$ & $179(31.74)$ & 37.2 & $<0.001$ & $3.022(2.02-4.53)$ \\
\hline Control $(n=17,487)$ & $537(7.64)$ & $801(7.66)$ & 0.0 & 0.952 & $1.003(0.9-1.11)$ \\
\hline \multicolumn{6}{|c|}{ Checked orthostatic blood pressure } \\
\hline Trained providers $(\mathrm{n}=783$ ) & $2(0.91)$ & $55(9.75)$ & 18.3 & $<0.001$ & $10.678(2.63-43.4)$ \\
\hline Control $(n=17,487)$ & $103(1.46)$ & $163(1.56)$ & 0.2 & 0.617 & $1.064(0.83-1.36)$ \\
\hline \multicolumn{6}{|l|}{ Referred to physical therapya } \\
\hline Trained providers $(\mathrm{n}=783$ ) & $86(39.27)$ & 237 (42.02) & 0.5 & 0.483 & $1.070(0.88-1.3)$ \\
\hline Control $(n=17,487)$ & $2960(42.09)$ & $4657(44.54)$ & 10.3 & 0.001 & $1.058(1.02-1.1)$ \\
\hline \multicolumn{6}{|c|}{$\begin{array}{l}\text { Note: This table shows provider practice patterns related to fall risk during PCP visits with older adult patier } \\
\text { score on the STEADI and in the } 12 \text { months pre/post training. } \\
\text { PCP, primary care provider; RR, relative risk; STEADI, Stopping Elderly Accidents, Deaths, and Injuries. } \\
\text { a Referral within } 14 \text { days after PCP visit. }\end{array}$} \\
\hline
\end{tabular}

PT. In comparing the 2 groups in the 12 months after training (Table 4), for their patients at risk of falling, the fellows were 4 times more likely to complete fall risk visits and over 6 times more likely to assess orthostatic blood pressure than their nontrained peers. Subgroup analysis of the $75+$ population revealed similar trends and significance, but these results are not included here.

\section{Discussion}

This study aimed to improve not only providers' knowledge and confidence in caring for older adults at increased risk to fall, but also their clinical practice in assessing and managing fall risk. In addition to improved knowledge and confidence, we found that the fellows increased their discussion of fall risk (through fall risk visits) and their assessment of orthostatic blood pressure for all of their patients, not just for those identified at increased risk to fall. This improvement held true for the fellows themselves before and after the intervention, but also as compared to their nontrained peers. These practice improvements for all of their 65+ patients, not just those identified as being at high risk to fall, are especially important, since studies indicate that early screening and intervention can help identify people at risk and prevent future falls. ${ }^{15}$

We were surprised that there were no significant differences in PT referrals made by the trained fellows, but this finding may have been confounded by the fact that the data included all PT referrals, regardless of diagnosis, not just those referrals that were fall-related. Furthermore, our baseline PT referral rates, at 39\% for the intervention group and $42 \%$ for the control group, are higher than national data when looking at rehabilitation use by older adults. ${ }^{26}$

In comparison to a study evaluating the occurrence of fall risk-related clinical practice in primary care before any fall-related educational intervention, orthostatics were checked less frequently in our study (10\% versus 30\%) and there were fewer PT referrals (42\%-44\% versus 53\%). ${ }^{27}$ However, the Phelan study took place in patients who had actually had a fall, rather than just having a higher risk for a fall, and was based on detailed chart review. Other studies $^{23,24}$ found higher rates of fall risk interventions, but did not break out PT referrals specifically.

In terms of the educational intervention itself, most studies of geriatric education interventions have measured 


\begin{tabular}{|c|c|c|c|c|c|}
\hline & Trained & Control & $\mathrm{X}^{2}$ & $P$ Value & RR (95\% CI) \\
\hline Providers & 5 & 46 & & & \\
\hline PCP visits within 60 days of $4+$ STEADI, post & 564 & 10,455 & & & \\
\hline Fall risk visits $(n=11,019)$ & 179 (31.74) & $801(7.66)$ & 382.8 & $<0.001$ & $4.143(3.61-4.76)$ \\
\hline $\begin{array}{l}\text { Checked orthostatic blood pressure } \\
(n=11,019)\end{array}$ & $55(9.75)$ & $163(1.56)$ & 185.2 & $<0.001$ & $6.255(4.66-8.39)$ \\
\hline $\begin{array}{l}\text { Referred to physical therapya } \\
(\mathrm{n}=11,019)\end{array}$ & $237(42.02)$ & $4657(44.54)$ & 1.4 & 0.240 & $0.943(0.85-1.04)$ \\
\hline \multicolumn{6}{|c|}{$\begin{array}{l}\text { Note: This table shows provider practice patterns related to fall risk during PCP visits with older adult pat } \\
\text { score on the STEADI and in the } 12 \text { months after the start of the training. } \\
\text { PCP, primary care provider; RR, relative risk; STEADI, Stopping Elderly Accidents, Deaths, and Injuries. } \\
\text { a Referral within } 14 \text { days after PCP visit. }\end{array}$} \\
\hline
\end{tabular}

changes in knowledge, confidence, or self-efficacy as they relate to geriatric competence, ${ }^{28-30}$ and do not measure practice change as an outcome outside of intent to change or self-reported practice change..$^{31,32}$ In general, practice change or longer-term health care-related outcomes have not been studied. Additionally, a range of dosages of educational interventions has been studied, from 1-hour lunchtime presentations ${ }^{23,32}$ to half-day ${ }^{29}$ or several half-day workshops, ${ }^{28}$ up to 160 hours over 10 months ${ }^{30}$ or 5 weekends over 6 months. ${ }^{31}$ The duration of our entire intervention at 160 hours over 6 months would be considered on the upper end of dosing relative to these studies, with our Mobility week intervention comprising 32 hours during 1 week. In the Warshaw study, despite 107 1-hour sessions being taught to over 60 physicians in 16 practices over 4 years, only 2 practices ultimately initiated any practice change projects. ${ }^{32}$ We believe that only curricula that embed practice change skills and opportunities, at a significant enough dose, can actually impact practice change in a sustainable manner.

Knowledge and skill acquisition among individual providers does not take place to a sufficient degree in the current health care arena, which is focused on productivity and short visit times. Consistent with other studies, we included interdisciplinary members of the primary care team for part of the mini-fellowship, although other studies used models that train across disciplines for the entirety of the learning experience. ${ }^{28-30,33}$ Our educational model was strengthened by including other professionals to provide some of the education and model the ideal geriatric team, including PT, occupational therapy, and pharmacy, for the week on mobility.

Most studies exploring interventions through geriatric educational initiatives are conducted within academic institutions, with a primary focus on physician faculty and, by extension, their teaching of residents and others. ${ }^{34,35}$ We believe our integrated model, which is steeped in community-based primary care practices like Lam's, ${ }^{31}$ offers the greatest outreach to large community-based care systems and their patients. Training providers to work with their teams to change their own practices first gives skills and expertise that help further establish them as geriatric champions within their practices, laying the groundwork for more widespread practice change at their clinics.

\section{Limitations}

In addition to the limitations described above relating to the capture of PT referrals, other limitations included the relatively short time period for follow-up data as well as the small size of the intervention group. However, we found value in the instructional depth that the small group size allowed.

While the nontrained providers did show some improvement during the same period, we believe the relative risk was not clinically significant. We suspect that the larger health system efforts to standardize screening of patients $65+$ across all clinics as a core quality metric confounded these results. The data analysis also included only fall-related patient visits that occurred with a provider who was that patient's PCP, which could have missed visits done by other PCP colleagues, RNs, or 
pharmacists in the same clinic, thus undercounting the true number of fall-related visits. Furthermore, counting of fall-related interventions relied upon providers documenting consistently in the EHR, which could also lead to under-represention of fall risk clinical efforts.

The data presented, while encouraging, do not reflect clinic-wide practice change patterns and are considered only proximate outcomes rather than more long-term or cost-related outcomes, as would be captured by fallrelated utilization measures like emergency room visits and hospitalizations. We expect to evaluate the broader impact and these value-based outcomes in the future. All providers and teams were from the same health care system, which may not allow our results to transfer to other organizations or regions of clinical practice.

\section{Summary}

This study demonstrates that an intensive mini-fellowship model of geriatrics training improved both knowledge and confidence in the realm of fall risk assessment and intervention among PCPs who had not been formally trained in geriatrics. More importantly, the training improved the fall-related care of their patients at increased risk to fall, but also of all of their older patients, with improvements in care measured up to a year after the mini-fellowship. Although this article only describes the work done as part of the Mobility aim of the 4M AFHS model, we believe the entire mini-fellowship curriculum offers the opportunity to "geriatricize" clinicians and their teams in learning geriatric principles and skills that they can translate into their practice in a sustainable way, as Tinetti encourages. ${ }^{8}$ Future study to evaluate other process outcomes more precisely, such as PT, as well as cost- and value-based outcomes, and the influence of trained providers on their clinic partners, will further establish the value proposition of targeted, disseminated, intensive geriatrics training of primary care clinicians as a strategy of age-friendly health systems as they work to improve the care of their older adults.

Acknowledgment: We are grateful for the dedication and hard work of the 2018 Geriatric Mini-Fellowship fellows at Providence Health \& Services-Oregon who made this article possible. Thanks to Drs. Stephanie Cha, Emily Puukka-Clark, Laurie Dutkiewicz, Cara Ellis, Deb Frost, Jordan Roth, and Subhechchha Shah for promoting the
AFHS work within their Providence Medical Group clinics and to PMG leadership and the fellows' clinical teams for supporting the fellows, the AFHS work, and their older patients.

Corresponding author: Colleen M. Casey, PhD, ANP-BC, Providence Health \& Services, Senior Health Program, 4400 NE Halsey, 5th Floor, Portland, OR 97213; colleen.casey@providence.org.

Financial disclosures: None.

doi:10.12788/jcom.0026

\section{References}

1. US Department of Health and Human Services. 2018 Profile of Older Americans. Administration on Aging. April 2018.

2. Roberts AW, Ogunwole SU, Blakeslee L, Rabe MA. The population 65 years and older in the United States: 2016. Washington, DC: US Census Bureau; 2018.

3. American Board of Medicine Specialties. 2017-2018 ABMS Board Certification Report. www.abms.org/media/257802/ abms-board-certification-report-2017-2018.pdf. Accessed November 3, 2020.

4. US Department of Health and Human Services, Health Resources and Services Administration, National Center for Health Workforce Analysis. National and regional projections of supply and demand for geriatricians: 2013-2025. Rockville, MD: US Department of Health and Human Services; 2007.

5. American Association of Nurse Practitioners, NP Facts: The Voice of the Nurse Practitioner. 2020. https://storage.aanp.org/www/ documents/NPFacts_080420.pdf.

6. Tinetti ME, Naik AD, Dodson JA, Moving from disease-centered to patient goals-directed care for patients with multiple chronic conditions: patient value-based care. JAMA Cardiol. 2016; 1:9-10.

7. Fried LP, Hall WJ. Editorial: leading on behalf of an aging society. J Am Geriatr Soc. 2008;56:1791-1795.

8. Tinetti M. Mainstream or extinction: can defining who we are save geriatrics? J Am Geriatr Soc. 2016;64:1400-1404.

9. Jafari P, Kostas T, Levine S, et al. ECHO-Chicago Geriatrics: using telementoring to "geriatricize" the primary care workforce. Gerontol Geriatr Educ. 2020;41:333-341.

10. Fulmer T, Mate KS, Berman A. The Age-Friendly Health System imperative. J Am Geriatr Soc. 2018;66:22-24.

11. Mate KS, Berman A, Laderman M, et al. Creating Age-Friendly Health Systems - A vision for better care of older adults. Healthc (Amst). 2018;6:4-6.

12. Tinetti ME, et al. Patient priority-directed decision making and care for older adults with multiple chronic conditions. Clin Geriatr Med. 2016;32:261-275.

13. Stevens JA, Phelan EA. Development of STEADI: a fall prevention resource for health care providers. Health Promot Pract. 2013;14:706-714.

14. Rubenstein $\mathbf{L}$, et al. Validating an evidence-based, self-rated fall risk questionnaire (FRQ) for older adults. J Safety Res. 2011;42:493-499.

15. Grossman DC, et al. Interventions to prevent falls in community-dwelling older adults: US Preventive Services Task Force Recommendation Statement. JAMA. 2018;319: 1696-1704.

16. Tricco AC, Thomas SM, Veroniki AA, et al. Comparisons of interventions for preventing falls in older adults: a systematic review and meta-analysis. JAMA. 2017;318:1687-1699. 
17. Gillespie LD, Robertson MC, Gillespie WJ, et al. Interventions for preventing falls in older people living in the community. Cochrane Database Syst Rev. 2012(9):CD007146.

18. Bergen G, Stevens MR, Burns ER. Falls and fall injuries among adults aged $\geq 65$ years - United States, 2014. MMWR Morb Mortal Wkly Rep. 2016;65:993-998.

19. Burns E, Kakara R. Deaths from falls among persons aged $>=65$ Years - United States, 2007-2016. MMWR Morb Mortal Wkly Rep. 2018;67:509-514.

20. Shankar KN, Liu SW, Ganz DA. Trends and characteristics of emergency department visits for fall-related injuries in older adults, 2003-2010. West J Emerg Med. 2017;18:785-793.

21. Hoffman GJ, et al. Posthospital fall injuries and 30-day readmissions in adults 65 years and older. JAMA Netw Open. 2019;2:e194276.

22. Eckstrom E, Parker EM, Shakya I, Lee R. Coordinated care plan to prevent older adult falls. 2018. Atlanta, GA: National Center for Injury Prevention and Control, Centers for Disease Control and Prevention; 2018.

23. Eckstrom E, Parker EM, Lambert GH, et al. Implementing STEADI in academic primary care to address older adult fall risk. Innov Aging. 2017; 1:igx028.

24. Johnston YA, Bergen G, Bauer M, et al. Implementation of the stopping elderly accidents, deaths, and injuries initiative in primary care: an outcome evaluation. Gerontologist. 2019;59:1182-1191.

25. Phelan EA, Mahoney JE, Voit JC, Stevens JA. Assessment and management of fall risk in primary care settings. Med Clin North Am. 2015;99:281-293.

26. Gell NM, Mroz TM, Patel KV. Rehabilitation services use and patient-reported outcomes among older adults in the United States. Arch Phys Med Rehabil. 2017;98:2221-2227.e3.
27. Phelan EA, Aerts S, Dowler D, et al. Adoption of evidence-based fall prevention practices in primary care for older adults with a history of falls. Front Public Health. 2016;4:190.

28. Solberg LB, Carter CS, Solberg LM. Geriatric care boot camp series: interprofessional education for a new training paradigm. Geriatr Nurs. 2019;40:579-583.

29. Solberg LB, Solberg LM, Carter CS. Geriatric care boot CAMP: an interprofessional education program for healthcare professionals. J Am Geriatr Soc. 2015;63:997-1001.

30. Coogle CL, Hackett L, Owens MG, et al. Perceived self-efficacy gains following an interprofessional faculty development programme in geriatrics education. J Interprof Care. 2016;30:483-492.

31. Lam R, Lee L, Tazkarji B, et al. Five-weekend care of the elderly certificate course: continuing professional development activity for family physicians. Can Fam Physician. 2015;61:e135-141.

32. Warshaw GA, Modawal A, Kues J, et al. Community physician education in geriatrics: applying the assessing care of vulnerable elders model with a multisite primary care group. J Am Geriatr Soc. 2010;58:1780-1785.

33. Solai LK, Kumar K, Mulvaney E, et al. Geriatric mental healthcare training: a mini-fellowship approach to interprofessional assessment and management of geriatric mental health issues. Am J Geriatr Psychiatry. 2019;27:706-711.

34. Christmas $\mathrm{C}$, Park $\mathrm{E}$, Schmaltz $\mathrm{H}$, et al. A model intensive course in geriatric teaching for non-geriatrician educators. $J$ Gen Intern Med. 2008;23:1048-1052.

35. Heflin MT, Bragg EJ, Fernandez H, et al. The Donald W. Reynolds Consortium for Faculty Development to Advance Geriatrics Education (FD AGE): a model for dissemination of subspecialty educational expertise. Acad Med. 2012;87:618-626.

\section{Call for Contributions}

JCOM is seeking submissions of original research and descriptive reports of quality improvement projects.

Original research submissions can include reports of investigations that address questions about clinical care or the organization of health care and its impact on outcomes.

Descriptions and evaluations of quality improvement efforts will be considered for JCOM's "Reports from the Field" section. The section features reports on actions being taken to improve quality of care. Such reports may include, but are not limited to, the following items:

- Motivation for the project, including the role of research findings or internal or external benchmarking data

- Setting/demographics

- Intervention/implementation process

- Measurements

- Results

- Similarities to other approaches studied, limitations, applicability in other settings, etc.

Approximate length is 4000 words. Papers submitted are sent for peer review. Decisions about manuscripts are made within 8 weeks of receipt. Information for authors may be found at www.mdedge.com/jcomjournal.

If you are interested in submitting a paper, contact:

Robert Litchkofski, Editor

rlitchkofski@mdedge.com
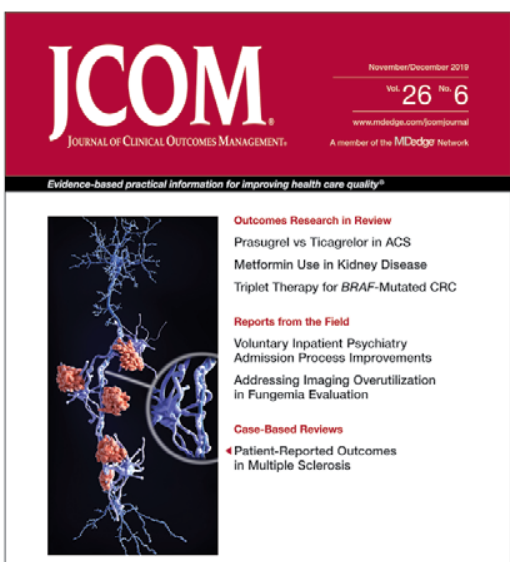